\title{
Segregation, quantum confinement effect and band offset for [110] SiGe NWs
}

\author{
Michele Amato', Maurizia Palummo ${ }^{2}$, and Stefano Ossicini ${ }^{*, 3}$ \\ ${ }^{1}$ CNR-Istituto di Nanoscienze "Centro $S^{3}$ " and Dipartimento di Fisica, Universita' di Modena e Reggio Emilia, \\ via Campi 213/A, 41125 Modena, Italy \\ ${ }^{2}$ European Theoretical Spectroscopy Facility (ETSF), CNR-INFM-SMC, Dipartimento di Fisica, Università di Roma, \\ 'Tor Vergata', via della Ricerca Scientifica 1, 00133 Roma, Italy \\ ${ }^{3}$ European Theoretical Spectroscopy Facility (ETSF), CNR-Istituto di Nanoscienze "Centro S 3 ", \\ Dipartimento di Scienze e Metodi dell' Ingegneria, and Centro Interdipartimentale "En\&Tech", \\ Università di Modena e Reggio Emilia, via Amendola 2 Pad. Morselli, 42100 Reggio Emilia, Italy
}

Received 15 September 2009, revised 25 February 2010, accepted 2 March 2010

Published online 22 June 2010

Kewords electronic structure, quantum confinement, $\mathrm{SiGe}$

*Corresponding author: e-mail stefano.ossicini@unimore.it, Phone: +39 0522 522211, Fax: +39 0522522312

Results of first-principles DFT simulations provide strong evidence that, at zero temperature, for [110] oriented SiGe nanowires (NWs), the segregated structure is favoured with respect to the mixed ones; for this observation two different schemes of calculations are presented and discussed. Moreover the segregation strongly influences the NWs electronic proper- ties, inducing a reduced quantum confined effect (RQCE). We show here that it depends on the effect of strain in the plane normal to the direction of growth and not on the choice of lattice parameter in the direction of growth. A qualitative evaluation of the band offset between $\mathrm{Si}$ and $\mathrm{Ge}$ for $\mathrm{SiGe} \mathrm{NWs}$ is also presented.
1 Introduction In recent years particular attention of world research has been devoted to group IV semiconductor nanowires (NWs) [1,2], a powerful class of nanostructures which is opening new substantial opportunities for nanoelectronics, nanophotonics and photovoltaics [3-7]. These nanostructures are one-dimensional materials with diameter from few to some tens of nanometers. They present unique size dependent electronic, optical and transport properties that are intrinsically associated with their low dimensionality and quantum confinement effect (QCE). In particular the SiGe NWs represent a class of NWs for which, recently, various experimental methods of synthesis have been developed $[2,8,9]$. In these one-dimensional systems the modulation of electronic properties can be reached not only by varying the size, like in pure $\mathrm{Si}$ or Ge NWs $[10,11]$, but also by changing the relative composition of $\mathrm{Si}$ and $\mathrm{Ge}$ and the geometry of interface of the two materials. For this kind of NWs much attention has been devoted to the coreshell geometry, for which useful applications such as highmobility FET and low temperature quantum devices have been demonstrated $[12,13]$. Nevertheless, there is a lack of theoretical efforts on SiGe NWs structural, electronic and optical properties. In a recent work [14], we have described the structural and electronic properties of SiGe NWs, whose lattice contains the same number of $\mathrm{Si}$ and $\mathrm{Ge}$ atoms but arranged in a different manner, as in Fig. 1. Our results demonstrate that the wires with a clear interface between $\mathrm{Si}$ and Ge form the most stable structure, show a particular QCE and present a clear separation between valence states and conduction states. This last property is strongly confirmed by the many body calculation which suggests that this type of wire, under optical excitation, displays a clear electron-hole separation, a property which can have relevant application for the developing of new solar cells, overcoming the present efficiency limits. Following the way of our previous work, in this paper we present first-principles calculations about [110] SiGe NWs, focusing the attention about the thermodynamical stability of these structures and on the role played by QCE and the strain on the electronic properties.

2 NWs geometries and calculation details The free standing NWs considered here are very-well faceted 

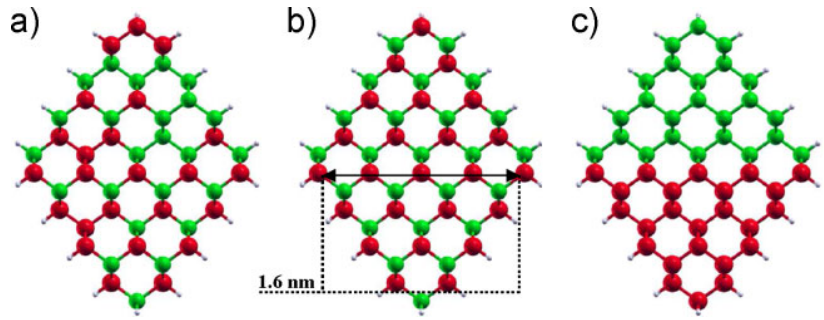

Figure 1 (online colour at: www.pss-b.com) Top view of SiGe Random (a), Mixed (b), Half (c) nanowires. Green spheres represent $\mathrm{Si}$ atoms, red spheres $\mathrm{Ge}$ atoms, while the small white spheres are $\mathrm{H}$ atoms used to saturate the dangling bonds.

nanostructures, are oriented along the [110] direction (the preferred growth direction at small diameter [15]) and have diameter (defined as in Fig. 1) ranging from 0.8 to $1.6 \mathrm{~nm}$; the way of constructing the input geometry of these wires is described in Ref. [14].

To eliminate the intra-gap states we saturate the surface of the wires with hydrogen atoms; we study pure Si NWs, pure Ge NWs and SiGe nanoalloys with fixed composition $\mathrm{Si}=0.5$ and $\mathrm{Ge}=0.5$. The top view of $\mathrm{SiGe}$ NWs are shown in Fig. 1: for these heterostructures we study three different homotops (i.e. NWs with the same composition, the same geometry, but different distribution of the two types of atoms in the unit cell); in particular we have considered Random, Mixed and Half NWs: in the first one (see Fig. 1a) the distribution of $\mathrm{Si}$ and $\mathrm{Ge}$ atoms in the unit cell is totally random [16, 17]; the second one (see Fig. 1b) represent a perfect $\mathrm{SiGe}$ alloy, in which every type of atom is bonded in a tetrahedral geometry with four atoms of the other type; for Half NWs (see Fig. 1c), instead, one half of the cross-section of the wire is made up of Si and the other one of Ge. All the calculations are performed in the framework of density functional theory (DFT) in the local-density approximation (LDA) $[18,19]$, using norm-conserving pseudopotentials as implemented in the ESPRESSO package [20]. The considered NWs are embedded in large supercells with enough vacuum space (more than $10 \AA$ ) to prevent interactions between the periodic replicas. An energy cutoff to $30 \mathrm{Ry}$ and a grid of $16 \times 1 \times 1$ points for the sampling of the Brillouin zone have been used. To take into account strain effects, for all the structures a full geometry optimization is performed. Concerning the size of the cell along the growth direction, the strain has been considered using Vegard's law [21, 22], which states that the relaxed lattice parameter of a two component system is a linear function of the composition; this is a very common procedure for semiconductor bulk alloys and recently it has been demonstrated that it is still valid for nanoalloys [23-26].

3 Formation enthalpy In Ref. [14] we have presented a set of calculations of formation enthalpy (FE) of SiGe NWs of Fig. 1 at different diameters and we have demonstrated two peculiar aspects: (i) Half NWs represent the most stable structure at any diameter (ii) this stability is improved increasing the diameter, so it has been possible to
Table 1 Formation enthalpy $\Phi^{*}$ (in eV) calculated with Eq. (1) for pure Si NWs (second column), pure Ge NWs (third column), Random NWs (fourth column), Half NWs (fifth column) and Mixed NWs (sixth column) at different diameters.

\begin{tabular}{lccccc}
\hline diameter $(\mathrm{nm})$ & $\mathrm{Si}$ & $\mathrm{Ge}$ & Random & Half & Mixed \\
\hline 0.8 & 0.0 & 0.0 & 0.031 & 0.053 & 0.167 \\
1.2 & 0.0 & 0.0 & 0.255 & 0.109 & 0.372 \\
1.6 & 0.0 & 0.0 & 0.663 & 0.144 & 0.649 \\
\hline
\end{tabular}

say that the segregation results in a gain of energy and, for larger diameter, it is favoured with respect to any form of mixing (these results can be deduced by Table 1). Here we analyze deeper these results and we make a comparison between two different ways of calculating the FE, showing how they give the same physical results, but one of them is more realistic, because is closer to the reality of the experiment. The FE can be calculated in the way proposed by Ferrando et al. [27]:

$$
\begin{aligned}
& \Phi^{*}\left(\mathrm{Si}_{n} \mathrm{Ge}_{m} \mathrm{H}_{l}\right)=E_{\mathrm{tot}}\left(\mathrm{Si}_{n} \mathrm{Ge}_{m} \mathrm{H}_{l}\right) \\
& -m \frac{E_{\mathrm{tot}}\left(\mathrm{Ge}_{N} \mathrm{H}_{l}\right)}{N}-n \frac{E_{\mathrm{tot}}\left(\mathrm{Si}_{N} \mathrm{H}_{l}\right)}{N},
\end{aligned}
$$

where $N=n+m$. In other words, in Eq. (1), one subtracts from the total energy of the nanoalloy, the appropriate fraction of the configurational energy of the pure reference $\mathrm{Si}$ and Ge NW. In this way $\Phi^{*}$ is unbiased, being 0 for the pure NWs (see Table 1).

Here we present the trend of FE scaling with the diameter, using (as other similar DFT studies on different nanosystems [28-32]) also the following formula for FE, introduced by Zhang and Northrup [33]:

$$
\begin{aligned}
& \Phi\left(\mathrm{Si}_{n} \mathrm{Ge}_{m} \mathrm{H}_{k}\right)=E_{\text {tot }}\left(\mathrm{Si}_{n} \mathrm{Ge}_{m} \mathrm{H}_{k}\right) \\
& \quad-m \mu\left(\mathrm{Ge}_{\text {bulk }}\right)-n \mu\left(\mathrm{Si}_{\text {bulk }}\right)-k \mu(\mathrm{H}),
\end{aligned}
$$

here $E_{\text {tot }}$ is the ground state total energy of a given $\mathrm{Si}_{n} \mathrm{Ge}_{m} \mathrm{H}_{l}$ NW and $\mu\left(\mathrm{Si}_{\text {bulk }}\right), \mu\left(\mathrm{Ge}_{\text {bulk }}\right)$ and $\mu(\mathrm{H})$ represent the total energy per atom calculated for the bulk of Si and Ge and for $\mathrm{a} \mathrm{H}_{2}$ molecule. The results, obtained using this formula, are shown in Fig. 2.

As we can see this formulation does not change the physical conclusions about the different $\mathrm{SiGe}$ systems (because the relative differences between the different $\mathrm{SiGe}$ systems remain the same): again the most stable SiGe NWs are the Half NWs, the segregation is favoured with respect to any form of mixing and this effect is strong for large wires. Nevertheless it must be pointed out that the use of Eq. (2) gives very different values for the FE of pure Si and Ge NWs, owing the fact that the total bulk energy per atom, of Si and $\mathrm{Ge}$, are very different. Consequently analyzing the trend of $\Phi$, going from the pure NW to a SiGe nanoalloy, a different behavior is obtained taking as reference the Ge NW or the $\mathrm{Si}$ NW. One could conclude that it should be more convenient, from a thermodynamic point of view, to synthesize a $\mathrm{SiGe}$ NW starting from a Ge NW rather than a Si NW. Clearly, 


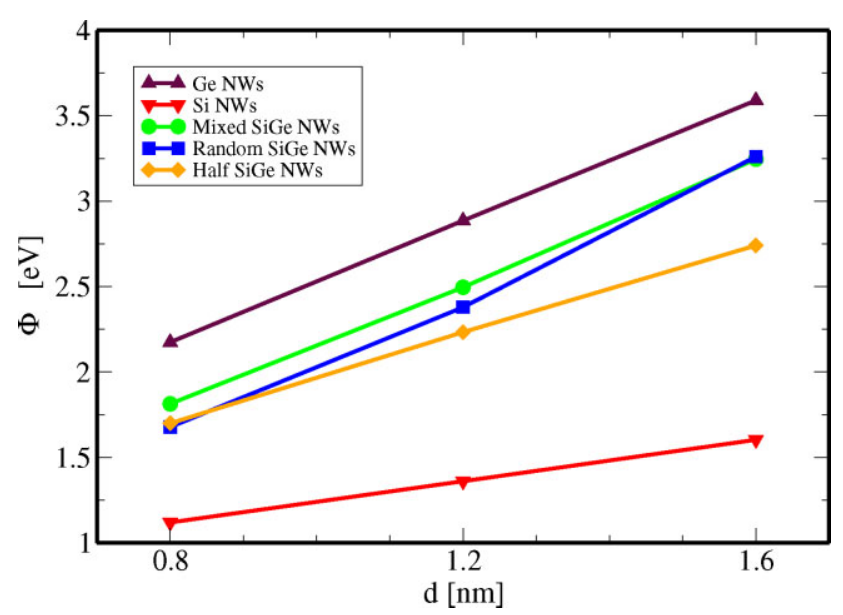

Figure 2 (online colour at: www.pss-b.com) Formation enthalpy $\Phi$ calculated with Eq. (2) for Random, Mixed and HalfSiGe NWs as function of their diameter. The same quantity for the corresponding pure $\mathrm{Si}$ or Ge NWs is also shown.

this conclusion is misleading and not realistic. In fact the comparison between a $\mathrm{SiGe}$ and a pure $\mathrm{NW}$ should give the same results if we take as reference a Si or a Ge NW. So, the use of Ferrando et al. formula, is in agreement with Zhang and Northrup formalism, but has the advantage that points out the FE in a very realistic mode which can be very useful for experimentalists. Observing the Table 1 we can draw the following conclusions: (i) All SiGe NWs FE have positive values with respect to that of pure $\mathrm{Si}$ and Ge NWs. By definition of FE, this condition indicates thermodynamical instability; consequently the decomposition into pure constituent crystals is favoured. (ii) An analysis of stability of SiGe NWs demonstrates that the segregation (i.e. the presence of a more ordered structure respect to a mixed or random one) at low temperatures, should be favoured with respect to any form of mixing, because lowers the FE. In our opinion the stability of Half NWs with respect to Random and Mixed NWs can be explained in the following way: in the Mixed configuration every $\mathrm{Si}$ atom (Ge atom) is bonded to four $\mathrm{Ge}$ atoms ( $\mathrm{Si}$ atoms), so the tetrahedral unit of the pure $\mathrm{Si}$ or $\mathrm{Ge}$ bulk is totally destroyed and the system needs a certain amount of energy to create new $\mathrm{Si}-\mathrm{Ge}$ bonds and to accomodate the new geometry of atoms (variation of bond lengths and bond angles); in the Half NWs this spending of energy is minimized, because the number of $\mathrm{Si}-\mathrm{Ge}$ bonds is minor, there are two bulklike zones in the wire (Si part and Ge part) in which the tetrahedral unit of the pure $\mathrm{Si}$ and Ge bulk is conserved and so the variation of geometry with respect to the bulk one is not so marked as in the previous case. The behavior of Random NWs is intermediate between the other two types of wires: in particular at small diameter it is close to Half NWs (at $0.8 \mathrm{~nm}$ the two values of practically coincide); this is due to the fact that when the diameter is small the number of possible configurations is low and so a random structure will be more segregated (i.e. more close to a Half $\mathrm{NW}$ ) rather than mixed (where there is not any form of segregation). On the contrary, at large diameter, the behavior of Random NWs is similar to that of Mixed NWs; now the number of possible configurations for a Random NW is larger, the probability for these type of wires to be segregated is lower and the FE increases. Probably, to analyze deeper this statement, it will be useful to separate the calculated FE into two parts, one related to the chemical energy of the alloy (connected with charge transfer, polarization or formation of new types of bonds) and the other one to the structural energy (connected with microscopic strain); we postpone this aspect to a future work.

4 Electronic properties It has been demonstrated that, for pure $\mathrm{Si}$ and pure Ge NWs [10,11], the QCE leads to an increase in the band gap with decreasing wire diameter and, thus, to a blueshift of the absorption and emission spectra; for SiGe core-shell NWs the band gap depends strongly on the relative composition of $\mathrm{Si}$ and $\mathrm{Ge}[25,34]$. We have demonstrated that Half $\mathrm{SiGe}$ NWs show a reduced quantum confinement effect (RQCE), which is more pronounced increasing the diameter of the wire, as shown in Table 2; this is related to a small opening of the gap [35].

We have ascribed the origin of this property to the different contribution to the QCE for $\mathrm{Si}$ and $\mathrm{Ge}$ and to the consequent type II staggered band-offset between $\mathrm{Si}$ and $\mathrm{Ge}$. In spite the fact that there are a lot of theoretical work which describes the band alignement for $\mathrm{Si} / \mathrm{Ge}$ superlattices and bulk heterojunctions [36-39], this effect at nanoscale is still under hot debate; in particular it is not still clear how the characteristic parameters of a bulk SiGe heterojunction [36] are affected by the QCE and by the strain and which of the two factors is predominant. In our previous calculations we have shown that the band offset derives from the different alignement of conduction and valence states for $\mathrm{Si}$ and $\mathrm{Ge}$ due to the different contribution of the two materials to the QCE; in that calculation we have taken into account the effect of strain performing a full relaxation af atoms in the plane normal to the growth direction and using the Vegard's law in the direction of growth. Here to analyze deeper how the strain affects the RQCE: we have performed full calculations for Half SiGe NWs using three different lattice parameters of (a) bulk Si, (b) bulk Ge and (c) use of Vegard's law. Figure 3 shows the variation in the electronic calculations (through the values of energy gaps) for SiGe Half NWs (with different diameters) induced by the choice of different lattice parameters in the direction of growth. The figure clearly shows that the effect of strain in the direction of

Table 2 DFT-LDA electronic gaps (in eV) for pure Si NWs (second column), pure Ge NWs (third column), Random NWs (fourth column), Half NWs (fifth column) and Mixed NWs (sixth column).

\begin{tabular}{llllll}
\hline diameter $(\mathrm{nm})$ & $\mathrm{Si}$ & $\mathrm{Ge}$ & Random & Half & Mixed \\
\hline 0.8 & 1.497 & 1.487 & 1.416 & 1.355 & 1.492 \\
1.2 & 1.155 & 1.120 & 1.111 & 0.8729 & 1.132 \\
1.6 & 0.987 & 0.938 & 0.909 & 0.599 & 0.954 \\
\hline
\end{tabular}




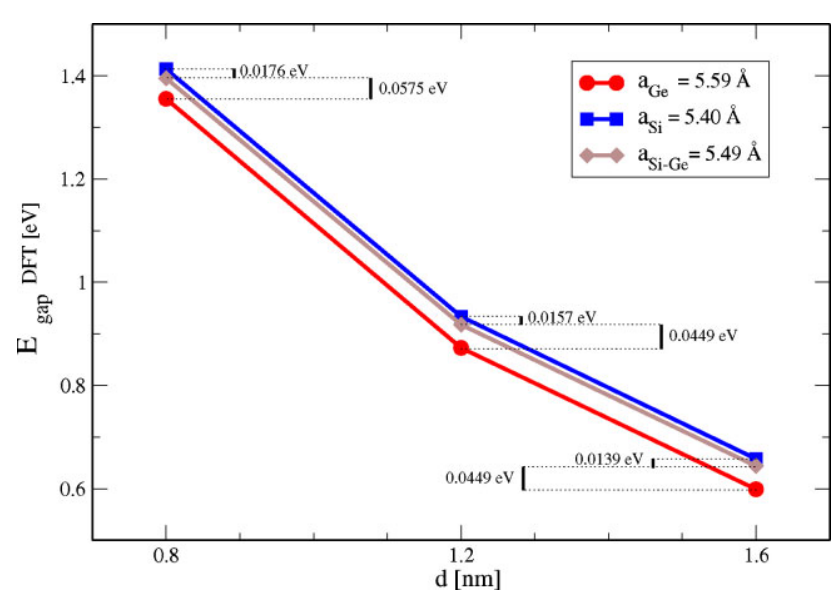

Figure 3 (online colour at: www.pss-b.com) DFT-LDA electronic gaps as a function of NW diameter, for Half NWs, with lattice parameter of bulk Si (blue line), bulk Ge (red line) and Vegard's one (brown line).

wire is very small in the band gap values and so it can be neglected; moreover, as expected, the result relative to the use of Vegard's law is intermediate between the results for bulk $\mathrm{Si}$ and bulk Ge, i.e. takes into account the effect of strain. So we can say that the RQCE for Half NWs is a property which is independent from the choice of the lattice parameter in the growth direction and is related to the geometry relaxation in its normal plane and to the different QCE of Si and Ge.

5 Evaluation of SiGe band offset for Half NWs The RQCE for Half NWs is related to the type II staggered band offset at the $\mathrm{Si} / \mathrm{Ge}$ interface, which comes out for the different QCE of Si and Ge and for the strain in the plane normal to the direction of growth. The role and the importance of semiconductor-semiconductor interfaces and junctions in modern electronic and optoelectronic is a crucial one and has been object of intense study in recent years. As mentioned before a lot of theoretical studies have analyzed and have evaluated the band offset at semiconductor interfaces for bulk heterojunction and superlattices [38-42]; nevertheless there is paucity of theoretical works which describe this phenomena at the nanoscale. In this section we try to give an evaluation of band offset between $\mathrm{Si}$ and Ge for Half NWs through a qualitative method which is not exact, but can give useful suggestions to understand the order of magnitude of band discontinuities. The band offset between two semiconductors is related to the band profiles at the interface, in particular to the valence and conduction discontinuities that accomodate the difference in the bandgap between the materials [41]; moreover at the nanoscale this phenomena can be affected by the different QCE of the two materials; the band offset between Si and Ge in Half NWs is a type II staggered one [37] and this implies that the valence band maximum (VBM) is located on one material and the conduction band minimum (CBM) on the other one. As
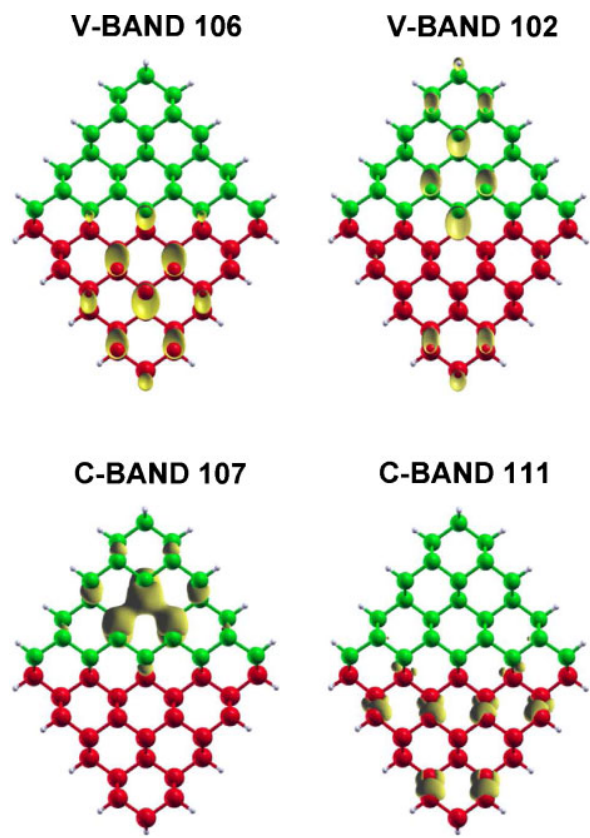

Figure 4 (online colour at: www.pss-b.com) Wave function localizations for Half $\mathrm{SiGe}$ NWs with $d=1.6 \mathrm{~nm}$; the first row reports valence states while the second one the conduction states. Green spheres represent $\mathrm{Si}$ atoms, red spheres Ge atoms, while the small white spheres are $\mathrm{H}$ atoms used to saturate the dangling bonds.

we have demonstrated in Ref. [14], for Half SiGe NWs the VBM is confined on the Ge part of the wire while the CBM on the Si part. To analyze the band profiles between $\mathrm{Si}$ and $\mathrm{Ge}$ for Half NWs we have examined all the electronic states near the valence and conduction band edges; to evaluate the valence and conduction band offsets (respectively, VBO and $\mathrm{CBO}$ ) we have taken the energy difference between the eigenvalue corresponding to the band edge (that are confined states) and the one of the first electronic state that is not confined; it is important to point out that this approach is only qualitative. We applied these criteria to the Half SiGe NW with $d=1.6 \mathrm{~nm}$ (this wire has 212 valence electrons, so the band 106 will be the VBM and the band 107 the CBM); in
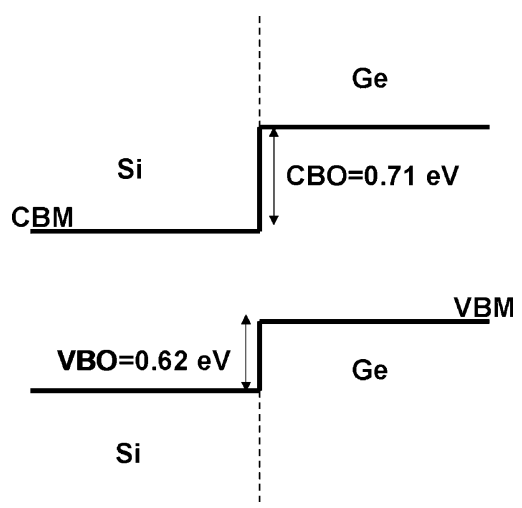

Figure 5 Band offset scheme for Half $\mathrm{SiGe}$ NWs with $d=1.6 \mathrm{~nm}$; the numerical estimation of $\mathrm{VBO}$ and $\mathrm{CBO}$ is also shown. 
Fig. 4 we report in the first row the VBM (v-band 106 of Fig. 4-completely located on the Ge part of the wire) and the first valence state not confined (v-band 102 of Fig. 4 - the first one not located on the Ge part of the wire), in the second row we have plotted the CBM (c-band 107 of Fig. 4 - completely located on the Si part of the wire) and the first conduction state not confined (c-band 111 of Fig. 4 - the first one not located on the Si part of the wire). So adopting these criteria the band offset can be presented schematically as in Fig. 5 with $\mathrm{VBO}=0.62 \mathrm{eV}$ and $\mathrm{CBO}=0.71 \mathrm{eV}$.

6 Conclusions In this paper we have characterized from a theoretical point of view in the DFT framework the thermodynamic and electronic properties of [110] SiGe NWs. The analysis of FE of these systems, presented with the Zhang and Northrup formalism, has confirmed the results of our recent work [14], pointing out that Half SiGe NWs, at zero temperature, represent the most stable structure with respect to the mixed ones; moreover it has pointed out how the Ferrando's approach is more suitable for the study of this type of nanoalloy. The calculations of the electronic properties of Half NWs have shown how the RQCE is due only to the different QCE of Si and Ge and to the strain in the plane normal to the direction of growth, and does not depend from the choice of the lattice parameter in the direction of growth. Finally we have given, through a qualitative approach, an evaluation of the band offset between $\mathrm{Si}$ and Ge for Half SiGe NWs with $d=1.6 \mathrm{~nm}$.

Acknowledgements We acknowledge MIUR-PRIN 2007, CINECA-CNR-INFM (for CPU time) and Ec e-I3 ETSF project (INFRA: no. 211956).

\section{References}

[1] Y. Cui and C. M. Lieber, Science 293, 851 (2001).

[2] L. Lauhon, M. S. Gudiksen, D. Wang, and C. M. Lieber, Nature 420, 57 (2002).

[3] B. Tian, X. Zheng, T. J. Kempa, Y. Fang, N. Yu, G. Yu, J. Huang, and C. M. Lieber, Nature 449, 885 (2007).

[4] G. Conibeer, M. Green, R. Corkish, Y. Cho, E. Cho, C. Jiang, T. Fangsuwannarak, E. Pink, Y. Huang, T. Puzzer, T. Trupke, B. Richards, A. Shalav, and K. Lin, Thin Solid Films 511, 654 (2006).

[5] A. I. Hochbaum, R. Chen, R. D. Delgado, W. Liang, E. C. Garnett, M. Najarian, A. Majumdar, and P. Yang, Nature 451, 163 (2008).

[6] Y. Huang, X. Duan, Y. Cui, L. Lauhon, K. Kim, and C. M. Lieber, Science 294, 1313 (2001).

[7] Y. Ahn, J. Dunning, and J. Park, Nano Lett. 5, 1367-1370 (2005).

[8] W. W. Fang, N. Singh, L. K. Bera, H. S. Nguyen, S. C. Rustagi, G. Q. Lo, N. Balasubramanian, and D. L. Kwong, IEEE Electron Device Lett. 28, 3 (2007).

[9] N. Singh, K. D. Buddharaju, S. K. Manhas, A. Agarwal, S. C. Rustagi, G. Q. Lo, N. Balasubramanian, and D. L. Kwong, IEEE Trans. Electron Devices 55, 11 (2008).

[10] M. Bruno, M. Palummo, A. Marini, R. Del Sole, V. Olevano, A. Kholod, and S. Ossicini, Phys. Rev. B 72, 153310 (2005).
[11] S. Beckman, J. Han, and J. Chelikowsky, Phys. Rev. B 74, 165314 (2006).

[12] J. Xiang, W. Lu, Y. Hu, Y. Wu, H. Yan, and C. M. Lieber, Nature 441, 489 (2006).

[13] Y. Hu, H. O. Churchill, D. J. Reilly, J. Xiang, and C. M. Lieber, Nature Nanotechnol. 2, 622 (2007).

[14] M. Amato, M. Palummo, and S. Ossicini, Phys. Rev. B 79, 201302(R) (2009).

[15] Y. Wu, Y. Cui, L. Huynh, C. J. Barrelet, D. C. Bell, and C. M. Lieber, Nano Lett. 4, 433 (2004).

[16] E. L. de Oliveira, E. L. Albuquerque, J. S. de Sousa, and G. A. Farias, Microelectron. J. 40, 762 (2009).

[17] The random geometry we consider is obtained by randomly substituting Si atoms with $\mathrm{Ge}$ ones in the plane normal to the direction of growth; as already done in other theoretical study [16] to make our comparison with other geometries we refer only to one random configuration, constructed in this way. We know that a correct and realistic description of a random structure should include and consider a very huge number of possible configurations and that our Random NWs, obtained with only one configuration, are not so close to the reality of the experiment; nevertheless our purpose in this paper is to describe how the effect of the disorder can modify the structural and electronic properties of the NWs with respect to Half and Mixed NWs; our aim is not to obtain a correct description of the properties of Random SiGe NWs (considering all the possible random configurations), but just to have one random configuration to make a comparison with the other types of geometries.

[18] M. Amato, M. Palummo, and S. Ossicini, Phys. Rev. B 80, 235333 (2009).

[19] For the inclusion of excitonic effects and self-energy corrections see the note in Ref. [18] and references therein.

[20] S. Baroni, A. Dal Corso, S. de Gironcoli, P. Giannozzi, C. Cavazzoni, G. Ballabio, S. Scandolo, G. Chiarotti, P. Focher, A. Pasquarello, K. Laasonen, A. Trave, R. Car, N. Marzari, and A. Kokaly, http://www.pwscf.org/.

[21] L. Vegard, Z. Phys. 5, 17 (1921).

[22] A. R. Denton and N. W. Ashcroft, Phys. Rev. A 43, 31613164 (2001).

[23] J. Yang, C. Jin, C. Kim, and M. Jo, Nano Lett. 6, 2679 (2006).

[24] S. J. Whang, S. J. Lee, W. F. Yang, and B. J. Cho, Appl. Phys. Lett. 91, 072105 (2007).

[25] R. Musin and X. Wang, Phys. Rev. B 71, 155318 (2005).

[26] L. Yang, R. N. Musin, X.-Q. Wang, and M. Y. Chou, Phys. Rev. B 77, 195325 (2008).

[27] R. Ferrando, J. Jellinek, and R. L. Johnston, Chem. Rev. 108, 845 (2008).

[28] E. Degoli, G. Cantele, E. Luppi, R. Magri, D. Ninno, O. Bisi, and S. Ossicini, Phys. Rev. B 69, 155411 (2004).

[29] D. V. Melnikov and J. R. Chelikowsky, Phys. Rev. Lett. 92, 046802 (2004).

[30] G. Cantele, E. Degoli, E. Luppi, R. Magri, D. Ninno, G. Iadonisi, and S. Ossicini, Phys. Rev. B 72, 113303 (2005).

[31] L. E. Ramos, E. Degoli, G. Cantele, S. Ossicini, D. Ninno, J. Furthmller, and F. Bechstedt, J. Phys.: Condens. Matter 19, 466211 (2007).

[32] F. Iori, E. Degoli, M. Palummo, and S. Ossicini, Superlattices Microstruct. 44, 337 (2008).

[33] S. B. Zhang and J. E. Northrup, Phys. Rev. Lett. 67, 2339 (1991).

[34] R. Musin and X. Wang, Phys. Rev. B 71, 165308 (2006). 
[35] The RQCE presented by Half SiGe NWs has crucial consequences on their optical behaviour: infact these wires show an enhanced optical efficiency at low energies (near infra-red region) with respect to the pure $\mathrm{Si}$ and pure Ge NWs; this property can be very relevant for various optoelectronic applications, in particular for solar cells. Our results about the optical features of these types of SiGe NWs compared with those ones of pure $\mathrm{Si}$ and pure Ge NWs will be the subject of a forthcoming paper.

[36] C. Van de Walle and R. Martin, J. Vac. Sci. Technol. B 3, 1256 (1985).
[37] C. Van de Walle and R. Martin, Phys. Rev. B 34, 5621 (1986).

[38] C. Van de Walle, J. Vac. Sci. Technol. A 21, S182 (2003).

[39] S. Ciraci and I. P. Batra, Phys. Rev. B 38, 1835 (1988).

[40] Baldereschi. Alfonso, S. Baroni, and R. Resta, Phys. Rev. Lett. 61, 734-737 (1988).

[41] M. Peressi, N. Binggeli, and A. Baldereschi, J. Phys. D, Appl. Phys. 31, 1273 (1998).

[42] C. Van de Walle and R. Martin, Phys. Rev. B 35, 8154 (1987). 